\title{
Criticality Aware Scheduler Prioritization in Virtual Orthogonal Multichannel Parallelism for 5G Cellular Network
}

DAN YE, Department of Computer Science \& Information Engineering, National Taiwan University

To fulfill various enhanced requirements of next generation wireless access, 5 G cellular network will drive towards higher energy efficiency, lower latency and higher reliable wireless networks. The key contributions can summarize as follows: (1) proposes frame-based max-weight scheduling (FMWS) with reconfiguration delay in combination of round-robin algorithm can dynamically control throughput and delay. The frame-based dynamic control (FBDC) policy is applicable to $5 \mathrm{G}$ cellular network control systems in data link layer, provides a new framework for developing throughput-optimal network control policies using state-action frequencies. (2) proposes a novel approach in MAC layer--Virtual multichannels Parallelism Carrier Sense Multiple Access (VMCP-CSMA) which can compute a set of TDDM schedules for multiple channels at once rather than computing one schedule at a time and constantly switching or recomputing schedules. (3) proposes a novel criticality aware scheduler prioritization in VMCP-CSMA policy can reorder a set of TDDM schedules based on max-weight scheduling with reconfiguration delay according to different application requirements. It can achieve high throughput and low delay with low complexity compared with other schedulings.

- Networks $\rightarrow$ Network layer protocols $\rightarrow$ Routing protocols - Networks $\rightarrow$ Network algorithms $\rightarrow$ Data path algorithms $\rightarrow$ Packet scheduling - Networks $\rightarrow$ Network algorithms $\rightarrow$ Control path algorithms $\rightarrow$ Network resources allocation $\bullet$ Networks $\rightarrow$ Network algorithms $\rightarrow$ Control path algorithms $\rightarrow$ Network control algorithms

Key words: 5G cellular network, maximal scheduling, throughput-optimal control, virtual multiple channels, criticality aware scheduler prioritization.

\section{INTRODUCTION}

Next generation wireless access will support a much wider range of use case characteristics and corresponding access requirements [1]. To guarantee support for latency-critical machine-type-communication [2] (MTC) applications, 5 G wireless access should allow for latencies on $1 \mathrm{~ms}$ or less. In the future, new paradigm in $5 \mathrm{G}$ cellular networks is full duplex cognitive radio with energy-efficient, low-latency and high-reliable. Promising network layer architecture of $5 \mathrm{G}$ will adopt SDN, NFV and control-data separation technique [3]. One of key enabling technologies in $5 \mathrm{G}$ Physical layer is Sparse Code Multiple Access (SCMA) [4], which could adapt the frame structure according to MAC payload. SCMA was proposed to allow complex entries in the signature matrix and each user enjoys a different codebook to avoid the coupling issues, which was considered as an energy efficient uplink approach for future $5 \mathrm{G}$ wireless systems. Other transmission technologies such as frequency domain (millimeter wave [5-6]) or spatial domain (massive MIMO technology [7]

D.Ye is with the Department of Computer Science and Information Engineering, National Taiwan

University, Taipei 106, Taiwan. E-mail: $\underline{\text { d03922001@esie.ntu.edu.tw }}$ 
or ultra dense network deployment) are to increase the radio resources. Cognitive radio (CR) network [8] is considered to be one of key promising components for next generation mobile network [7]. Interference-tolerant $\mathrm{CR}$ networks can achieve enhanced spectrum utilization by opportunistically sharing radio spectrum resources with licensed users, as well as better spectral and energy efficiency. 5G cellular network is developed towards energy-efficient [9], low latency and high reliable high mobility communication network.

With future deployment of Full duplex (FD) [10] systems, time division duplex (TDD) and frequency division duplex (FDD) will be harmoniously integrated, supporting all the half duplex mobile phones efficiently, leading to a much enhanced $5 \mathrm{G}$ system performance. The maximal scheduling algorithm for multiradio multichannel (MR-MC) [11] wireless networks can obtain optimal throughput control and extend local pooling factor to analyze capacity efficiency in the tuple-based greedy maximal scheduling (GMS) in MR-MC networks. To guarantee high throughput and optimal capacity efficiency, the Tuple-based Dynamic Distributed Maximal Scheduling (TDDMS) algorithm is developed for contiguous enhanced services in $5 \mathrm{G}$ communication to fulfill these significant performance requirements. This paper proposes a novel criticality aware scheduler prioritization in virtual multichannel parallelism algorithm for future 5 G systems.

The reminder of paper is organized as follows. Section II proposes criticality aware scheduler prioritization in virtual multichannel parallelism algorithm for 5 G cellular network. Section III describes system model, algorithm description and implementation of CASP-VOMCP, Coordinated criticality-Aware Rate-function Acceleration (CARA) as well as performance metrics of proposed CASP-VOMCP. Section IV derives an upper bound and lower bound on the rate-function. Simulation results on throughput and capacity are presented in section V. Finally, conclusions are reiterated in section VI.

\section{CRITICALITY AWARE SCHEDULER PRIORITIZATION IN VIRTUAL MULTICHANNEL PARALLELISM ALGORITHM FOR 5 G CELLULAR NETWORK}

\subsection{Variable Frame-based Max-Weight scheduling algorithm (VFMW)}

Time-varying channels and reconfiguration delays significantly reduces the system stability region and changes the structure of optimal policies. Framebased Max-Weight scheduling algorithm [12] that sets frame durations dynamically, as a function of the current queue lengths and average channel gains, is throughput-optimal. Characterizing the stability region of such system using state-action frequencies are stationary solutions to a Markov Decision Process (MDP) formulation. A dynamic control policy using the state-action frequencies and variable frames whose lengths are functions of queue sizes have proposed in [12] and show that it is throughput-optimal. The frame-based dynamic control (FBDC) policy is applicable to many network control systems, provides a new framework for developing throughput-optimal network control policies using state-action frequencies. Myopic policies are easy to implement and have better delay properties than FBDC. The network controller is to 
dynamically decide to stay with the current schedule of activations or to reconfigure to another schedule based on the channel process and the queue length information, where each decision to reconfigure leaves the network idle for an arbitrary but finite amount of time, corresponding to the reconfiguration delay. FBDC policy based on state-action frequencies achieves the full stability region. Myopic policies may achieve the full stability region while providing better delay performance than FBDC policy for most arrival rates.

\subsection{Optimal dynamic control policy with reconfiguration delays}

The important task is the analysis of reconfiguration delays on system stability and throughput-optimal max-weight scheduling algorithm. In a parallel queuing system with randomly varying connectivity, characterizing the stability region of the system uses throughput-optimality of the longest-connected-queue scheduling policy. Variable-size Frame-based Max-Weight (VFMW) [12] policy provides a good balance by dynamically adapting the frame length based on the queue sizes and stabilizes the system whenever possible, while providing a delay performance that is similar to that of a Max-Weight policy with fixed frame sizes (FFMW) policy with a small frame length for small arrival rates. Scheduling under reconfiguration delays and time-varying channels call for novel control algorithms that take advantage of the channel memory to improve performance. Joint scheduling [13] and high-throughput routing [14] in multihop delay tolerant networks (DTNs) with time-varying channels and reconfiguration delays are essential for dynamic control-optimal policy by cross-layer optimization.

\subsection{Delay-based server-side-greedy policy}

Delay-based Server-Side-Greedy (D-SSG) [15] guarantees a near-optimal ratefunction, delay performance to the rate-function, delay-optimal policy. D-SSG allocates the available server with smallest index. Delay weighted matching (DWM) [15] which maximizes the sum of the delay of scheduled packets in each time-slot. Not only throughput-optimal, but also rate-function delay-optimal maximizing the delay rate-function, rather than queue-length rate-function. The derived rate-function of DWM develops a simple threshold policy for admission control when the number of users scales linearly with the number of channels in the system. There are fixed integer delay threshold $b$ and maximum achievable rate-function for threshold $b-1$.

However, DWM incurs a high complexity $\mathrm{O}\left(\mathrm{n}^{5}\right)$. Hybrid scheduling uses Maximum-weight matching in the first stage. Low-complexity greedy policy achieves both throughput optimality and rate-function near-optimality. D-SSG allocates servers one by one to serve a connected queue that has the largest headof-line (HOL) delay. Greedy Frame-Based Scheduling (G-FBS) policy can exploit a key property of D-SSG. G-FBS policy guarantees a near-optimal rate-function, that D-SSG dominates G-FBS in every sample-path. D-SSG has a low complexity $2 \mathrm{n}^{2}+2 \mathrm{n}$. The queue-length-based scheduling Q-SSG has a positive queue-length rate-function for restricted arrival processes that not only across users, but also in time. D-SSG not only guarantees a near-optimal rate-function, but also has a 
similar delay performance to the rate-function delay-optimal policy.

\subsection{Achieving optimal throughput utility and low delay with CSMA algorithm by a virtual multichannel approach}

Virtual-Multi-Channel CSMA (VMC-CSMA) [16] can dramatically reduce delay. Virtual-Multi-Channel CSMA (VMC-CSMA) is to use multiple virtual channels and compute a good set of feasible schedules simultaneously without switching or recomputing schedules. In contrast, CSMA algorithm has large delay that may grow exponentially with the network size, which does not fulfill the requirement of low delay in most applications. VMC-CSMA algorithm can achieve both high throughput utility and low delay with low-complexity. Both the expected packet delay and the tail distribution of the head-of-line (HOL) waiting time at each link can be bounded independently of network size.

The main novelty of the proposed algorithm to significantly reduce delay is to take advantage of multiple physical or virtual channels. Suppose that there are two separate channels for the same topology and each has half the bandwidth of the original system. If one channel uses the odd schedule, the other channel uses the even schedule. Each link can achieve both target rate of 0.5 as well as low delay. The key idea is to compute a set of good schedules for multiple channels at once rather than computing one schedule at a time and constantly switching or recomputing schedules. There is $\mathrm{C}$ virtual channels. Each virtual channel can have a different schedule. By randomly choosing a virtual channel and using the corresponding schedule at each time-slot, then emulate the multiple physical channels. VMC-CSMA algorithms iteratively update the schedules across all virtual channels to optimize the total system utility. VMC-CSMA only requires local information exchange and incurs a low complexity that increases linearly with the number of virtual channels $\mathrm{C}$. The VMC-CSMA algorithm achieves both high throughput and low delay which does not grow with the network size with low complexity.

\section{CRITICALITY AWARE SCHEDULER PRIORITIZATION IN VIRTUAL ORTHOGONAL MULTI-CHANNEL PARALLELISM (CASP-VOMCP)}

This section is to describe system model, algorithm description and implementation of CASP-VOMCP, Coordinated criticality-Aware Rate-function Acceleration (CARA) as well as performance metrics of proposed CASP-VOMCP, then to derive an upper bound and lower bound on the rate-function that can be achieved by any scheduling policy. Furthermore, conduct numerical simulations and present results on throughput optimality and near-optimal rate-function for low-complexity greedy policy.

\subsection{System model}

Wireless system with $\mathrm{N}$ users is a multichannel system with only one physical channel, $\mathrm{C}$ virtual channels and computing multiple feasible schedules simultaneously. In each time-slot, a virtual channel can be allocated only to one user, but a user can be allocated with multiple virtual channels simultaneously. Multiple different feasible schedules group into one cluster and execute simultaneously in multiple parallelized virtual channels. It can quickly adapt to 
network traffic changes. Assuming that the number of users is equal to the number of virtual channels, the admission control needs to be carefully designed if the number of users becomes too large.

Let Qi denote FIFO queue associated with the ith user, let $\mathrm{Sj}$ denote jth server. Unit channel capacity, at most one packet from Qi can be served by Sj. Let $C_{i, j}(\mathrm{t})$ denote the connectivity between queue $\mathrm{Qi}$ and server $\mathrm{Sj}$ in time-slot $\mathrm{t}$. $C_{i, j}(\mathrm{t})$ can be modeled as a Bernoulli random variable with a parameter $q \in(0,1)$

$$
C_{i, j}(\mathrm{t})=\left\{\begin{array}{l}
1, \text { with probability } \mathrm{q} \\
0, \text { with probability } 1-\mathrm{q} .
\end{array}\right.
$$

Different application requirement employ different schedulers, different schedulers configure different weight values. The higher connectivity it has, the larger weight value the scheduler has. The larger weight values, the higher priority the scheduler has. The more criticality, the higher priority different schedulers have. Different network size determines different number of channels. In multichannel OFDM system, the key challenges are how to design efficient scheduling policy that can simultaneously achieve high throughput and low delay, high data rates, lower latency and much better user experience and how to design high-performance scheduling policies at a low complexity.

In OFDM-based LTE systems [17], the transmission time interval (TTI), within which the scheduling decisions made is only $1 \mathrm{~ms}$. Assuming that a singlecell multichannel system consisting of $\mathrm{C}$ virtual orthogonal channels and a proportionally large number of users, with intermittent connectivity between each user and each channel. The base station maintains a separate first-in-firstout (FIFO) queue, round robin queue, WFQ queue associated with each user, which buffers the packets in the data link layer.

In VMC-CSMA policy, by randomly choosing a virtual channel and using the corresponding schedule at each time-slot, then emulate the multiple physical channels. Nevertheless, this paper proposes CASP-VOMCP which chooses multiple virtual channels in parallel access based on criticality aware scheduler priority. CASP-VOMCP only requires local information exchange and incurs a low complexity that increases linearly with the number of virtual channels $\mathrm{C}$. The throughput and delay performance of CASP-VOMCP is with respect to C. Let $\mathrm{L}$ denote the total number of links. Moreover, under the assumption of logarithmic utility functions, the delay of each link will not grow with the network size. The delay [18] is defined as the time from packet arrival to the time when the packet is served at the MAC layer.

$$
\vec{V}=\left[\mathrm{V}_{l 1}, \ldots, \mathrm{V}_{l C}\right] \text { denotes schedule in all virtual channels, where } \mathrm{V}_{l k}=1 \text { if }
$$


link $l$ is scheduled in the $\mathrm{k}$-th virtual channel, and $\vec{V}=\left[\overrightarrow{\mathrm{V}}_{l}\right]$ denote the global schedules of all virtual channels and all links. The total number of virtual channels used by link $l$ is given by $x_{l}(\vec{V})=\sum_{k=1}^{C} V_{l k}$. At each time-slot t, the network chooses a virtual channel $k(t)$ from 1 to $\mathrm{C}$. All links in the network use the feasible schedule $\vec{S}(\overrightarrow{\mathrm{V}})_{k(t)}$ in this time-slot, each link $l$ transmits a packet if $\mathrm{V}_{l, k(t)}=1$. Each link only needs to know its own schedules $\vec{V}_{l}$. The long-term average rate [19] of link $l$ is $r_{l}(\vec{V})=\mathrm{x}_{l}(\vec{V}) / \mathrm{C}$, which is equal to actual transmission with probability. The average interservice time is $1 / r_{l}(\vec{V})$. For high throughput utility and low delay, the following optimization problem needs a solution.

$$
\max _{\vec{V}} \sum_{l=1}^{L} U_{l}\left(r_{l}(\vec{V})\right)
$$

\subsection{Coordinated criticality-Aware Rate-function Acceleration (CARA)}

CARA consists of three major components: (1) a dynamic scheduler cluster criticality predictor, (2) a greedy criticality-aware cluster scheduler, and (3) a criticality-aware virtual memory prioritization technique. Figure 1 illustrates the different components in CARA in the context of a cognitive radio. CARA identifies lower-delay scheduler clusters that have a high likelihood to become critical cluster at each time slot t. Similarly, CARA identifies higher-throughput scheduler clusters that have a higher priority to become critical cluster. CARA identifies faster-running scheduler clusters that have a higher probability to become more critical scheduler clusters. Cluster criticality CARA allocates more

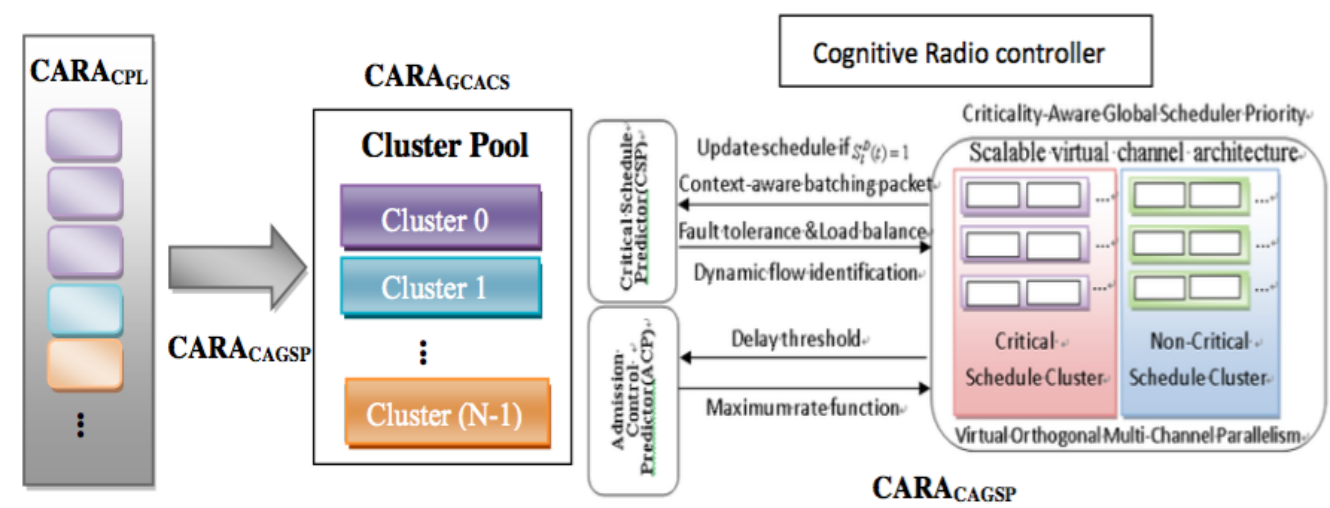

Figure 1. Different components in CARA in the Greedy Criticality-Aware Cluster Scheduler (GCACS) 
power and radio resource such as channel bandwidth, memory buffer to the context of cognitive radio network predicted-to-be-critical clusters with higher scheduling priorities. To reduce critical scheduler cluster execution time by allocating more power, channel bandwidth, the number of virtual channels, memory buffer, CARA proactively reserves a certain amount of memory capacity for data flow that is useful to critical scheduler clusters. CARA ensures an optimal capacity region for the critical scheduler clusters by reducing the delay.

3.3 Critical Cluster Identification with Criticality Prediction Logic (CPL)

In addition to updating the per-cluster criticality counters based on dynamic execution progress, CPL records additional delay experienced due to shared resource contention as well as scheduler delays, while updating the criticality counter. CPL monitors the service delay time between the current and the next packet delivery for each cluster and increment the criticality counter accordingly for all clusters. Table I presents Algorithm 1 the criticality counter update mechanism based on service delay time in CPL.

The per-cluster criticality counter is updated as follows:

$$
n \text { Criticality }=n \text { Schedule } * \text { w.ExeTimavg }+ \text { nDelay; }
$$

where $n$ Criticality represents the value of the per-cluster criticality counter, $n S c h e d u l e$ represents the relative schedule count disparity between the parallel clusters, w.ExeTimavg represents the per-cluster average execution time, and nDelay is the delay time incurred by shared resource contention, network congestion, and the scheduler, which is the time from packet arrival to the time when the packet is served at the MAC layer.

With the critical cluster identified by CPL, GCACS is designed to give more compute resources to critical clusters by prioritizing the execution of critical clusters and by providing a larger time slot to clusters in a greedy manner. GCACS selects a ready cluster for execution based on the degree of cluster criticality determined by the per-cluster criticality counter in CPL. If there are multiple clusters have the same criticality, the cluster scheduler will select the oldest one based on the Greedy-Then-Oldest algorithm. Then GCACS greedily executes schedules from the selected critical cluster until this particular cluster has no further available schedules. Consequently, the critical cluster not only receives a higher scheduling priority but also benefits from a larger time slot.

\subsection{Criticality-Aware Global Scheduler Priority (CAGSP)}

In addition to allowing the critical clusters to access parallelized virtual channels more often and for longer time duration, CAGSP is designed to allocate a dynamic data flow that will be used by the critical clusters for performance guarantee. Critical clusters are delay-critical and should be treated with higher priority. Schedule Clusters with the lower delay should be assigned with higher priority. Criticality-Aware Task Scheduler (CATS) uses bottom-level longest-path priorities. CAGSP first predicts critical schedule-schedule that will be used by critical clusters-with the critical schedule predictor, and retains these critical schedule in the schedule cluster partition reserved for critical clusters. 


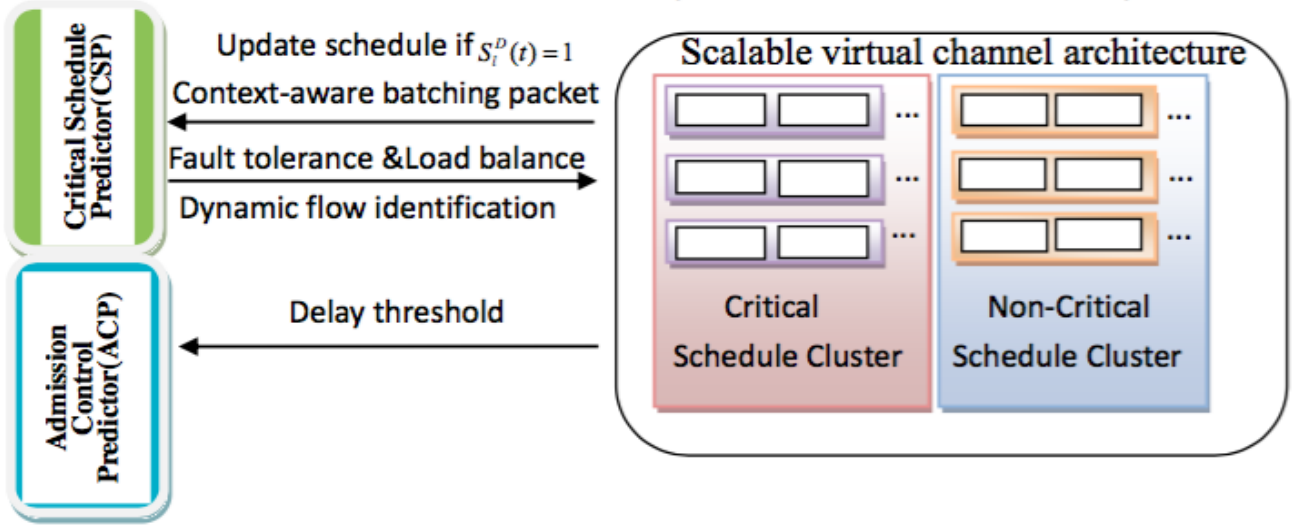

Figure 2. Criticality Aware Scheduler Prioritization in Virtual Orthogonal Multi-Channel Parallelism (CASP-VOMCP)

Figure 2 shows the proposed CAGSP scheme and interface between the different components. Schedules partition for cluster criticality. CAGSP partitions the schedules into two parts in the granularity of ways: critical schedule clusters and non-critical schedule clusters. Dynamically tune the size of the critical and non-critical schedule cluster partitions based on the CAGSP.

Critical schedule predictor (CSP) differentiates critical schedules from noncritical schedules such that an incoming schedule will be reserved for critical or non-critical schedules. Admission control predictor (ACP) learns and predicts the number of available virtual channels and the number of feasible schedules as well as the priority, the delay of scheduler. The benefit of ACP is virtualization-based high-speed packet delivery. Virtual Orthogonal Multi-Channel Parallelism (VOMCP) constructs a memory sharing framework for network data which can achieve efficient packet processing and higher concurrency. Memory sharing framework can facilitate high speed inter-virtual channels communication. Scalable virtual channel architecture is depicted in Figure 2.

\subsection{CASP-VOMCP Algorithm Description and Implementation}

CASP-VOMCP updates the global schedule $\vec{V}$ iteratively over time, which depends on the set $\mathrm{S}$ of decision schedules in local schedules. Define a set $\mathrm{S}$ of decision schedules satisfies the following three conditions.

A1) Each decision schedule is a feasible schedule. Each link $l$ must be scheduled by one decision schedule in $\mathrm{S}$.

A2) Each decision schedule is made on criticality-aware global scheduler priority.

A3) Each link scheduled by a decision schedule can broadcast $\mathrm{C}$ bits to its neighbors in a time-slot.

CASP-VOMCP algorithm:

Decision Phase: Choose a decision schedule $\vec{S}^{M P}(\mathrm{t})=\left[\mathrm{S}_{l}^{M P}(\mathrm{t})\right]$ at each time $\mathrm{t}$. 
MP denotes MaxPriority.

Update Phase: for each link 1 , if $\mathrm{S}_{l}^{M P}(\mathrm{t})=0$, let $\vec{V}_{l}^{M P}(\mathrm{t})=\vec{V}_{l}^{M P}(\mathrm{t}-1)$. Otherwise, $\mathrm{S}_{l}^{M P}(\mathrm{t})=1$ and $\mathrm{S}_{l^{\prime}}^{M P}(\mathrm{t})=0$ for every link $l^{\prime} \in \varepsilon_{l}$, perform Algorithm 2 in Table II.

Scheduling Phase: A common virtual channel $\mathrm{k}(\mathrm{t})$ is chosen by all links in the 5 G cellular network if $\vec{V}_{l, k(t)}^{M P}(\mathrm{t})=1$ each link 1 transmits a packet. Perform Algorithm 3 in Table III. Table III presents Algorithm 3 the process of CARA with three major components.

Congestion Control: (1) Context-aware admission control: accelerate data processing and alleviate congestion. There are fixed integer delay threshold $b$ and Maximum achievable rate-function for threshold b-1. (2) Throughput-based congestion control: jointly adjusted to control throughput on incast congestion. (3) Rate-based congestion control: decrease packets sending rate by half. (4) Distribution-based congestion control [15]: each link 1 injects a random number of packets according to a Poisson distribution with mean

$$
r_{l}=\arg \max _{r \geq 0}\left\{\mathrm{U}_{l}(\mathrm{r})-\beta \mathrm{Q}_{l}(\mathrm{t}) \mathrm{r}\right\} \text { where } \beta>0 .
$$

These prioritization schedulers are performed dynamically and potentially in parallel in different virtual channels simultaneously. While some schedulers are being prioritized, others assigned to available virtual channels to execute. Figure 3 shows a scheme of the operation of the Criticality-Aware Global Scheduler Priority (CAGSP).The number inside each node is the bottom level of the schedule: the length of the longest path from this node to node with rank 0 . The priority of a schedule is given by its bottom level. The number outside each node is the schedule id which is used in the text to refer to each schedule. Critical schedules

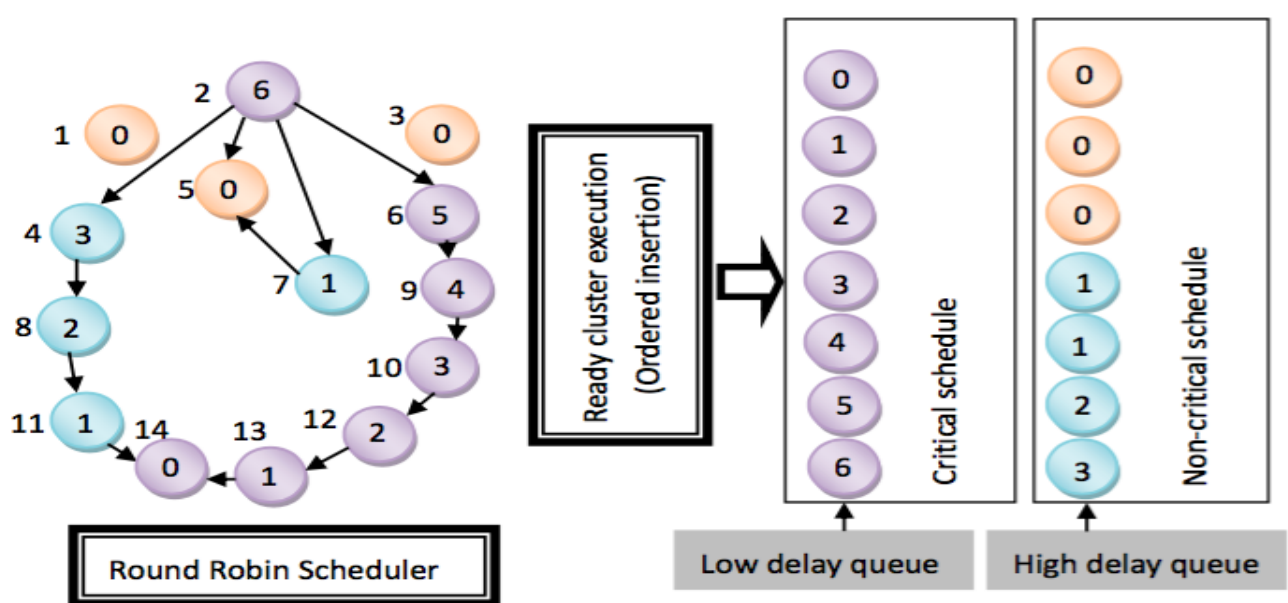

Figure 3. Round Robin Schedule prioritization with CATS. Nodes are marked with the bottom level of each scheduler. Pattern-purple nodes mark the critical schedulers with highest priority which processed in low delay queue. The blue nodes and orange nodes mark non-critical schedulers with low priority. 
are inserted in the critical queue, and non-critical schedules to the non-critical queue. The insertion is ordered with the highest priorities at the head of the queue and the lowest priorities at the tail.

Clustering schedules first separate schedules into clusters, where each cluster is to be executed on the different queues. During the clustering stage, assume an unlimited number of available queues. If the number of clusters exceeds the size of memory space, merging stage joins multiple clusters or sharing memory framework so that they can match the requirement of memory space. The heuristic clusters schedules that can execute in parallel according to their connectivity $\mathrm{C}(i, j)$ between queue $\mathrm{Qi}$ and server $\mathrm{Sj}$ and assigns priorities to the schedules in a cluster according to its execution time, schedules with the highest execution time have the highest priority. The scheduler enables dynamic scheduling of multiple-sized schedules for heterogeneous systems. CASP-VOMCP allows multiple implementations of a given schedule to be executed on different virtual channels. The scheduler assigns the execution of a schedule to the best resource according to its earliest finish time.

Scheduling heuristics focus on the critical schedules to reduce total execution time [20]. To identify the schedules in the critical clusters, the rank of schedule is the maximum sum of computation and communication cost of the schedules from the packet arrive to packet is serviced. Schedule queue maintains a list of schedules sorted in decreasing order of their rank. CASP-VOMCP assigns the schedule with the highest rank to the cluster that finishes the execution of schedule at the earliest time. The schedule with the highest rank belongs to the critical cluster. It dynamically updates the computation and communication costs on multiple schedules of multiple clusters. On each step, these schedules are dynamically assigned to the clusters that minimize the execution time of critical cluster. When a decision schedule becomes ready, all critical schedules finished their execution, it is assigned to a ready queue [21]. The schedule is inserted in the corresponding ready queue: schedules in the critical cluster ready queue will be executed firstly, and tasks in the non-critical cluster queue will be executed later. When a cluster becomes idle, it tries to retrieve a schedule from its corresponding ready queue to execute.

Each cluster has a list to include its finished schedules (plist) [22]. The priority given to a schedule is the bottom level of the schedule. Table IV Algorithm 4 shows the schedule prioritization. Using plist for each schedule, update the priority on each ready schedule and finished schedule. If the plist of the current task (currPred) is empty, all the ready schedules have finished execution. If the priority of the current schedule (currPred) remains unchanged, which means that successor schedule (succ) does not belong to the longest path because its predecessor already has a higher priority (bottom level). The longest path starts from the schedule with maximum bottom level. At runtime, the longest path changes as schedules complete execution and new schedule are created. CAGSP manages to detect changes and dynamically decide if the ready schedule belongs to the longest path. 
To determine the criticality of a schedule [23], CAGSP keeps track of last discovered critical schedule. Then for each schedule that becomes ready, CAGSP checks the following conditions: (1) whether the priority of the current ready schedule is higher than the priority of the last discovered critical schedule (maxPriority). (2) Whether the current ready schedule is the highest-priority immediate successor of the last discovered critical schedule.

Scheduler can be marked as critical schedules with priority higher or equal to the priority of the last critical schedule. Table V shows Algorithm 5 Criticality Aware Scheduler Prioritization algorithm. The variable maxPriority is used to store the priority of the last critical schedule, and maxPrioritySchedule is used to store the last critical schedule. Initially, maxPriority is set to 1 and maxPrioritySchedule is set to NULL. On the first ready schedule, if its priority is higher or equal than 1 , it is considered to be the first schedule of the longest path. It is inserted in the critical queue and the variables maxPriority and maxPrioritySchedule are updated accordingly to determine correctly the criticality of the next ready schedule. In the rest of cases the schedule is not considered critical and it is inserted in the non-critical queue. If the priority of the ready schedule is equal to maxPriority -1 , and if it belongs to the successors of the schedule with the maximum priority and to the longest path, the schedule is determined to be critical, it is inserted in the critical cluster queue and the variables maxPriority and maxPrioritySchedule are updated.

Figure 4 depicts how to execute CAGSP. The hollow nodes are schedules that have finished execution and purple nodes are critical schedules. The number insider the nodes indicate their priority and the number outside the nodes show the schedule id, which is assigned in schedule creation order. The variable maxPriority corresponds to the priority of the last critical schedule and the maxScheduleSucc is the list of the successors the last Schedule, filled with the schedule ids of the successors. Initially, max-Priority is set to 1 and maxScheduleSucc is empty. When schedule 2 is about to be ready, it is inserted in the critical queue because its priority is higher than the maximum, which at the beginning is 1 . Then, the value of maxPriority is set to 6 (priority of schedule 2 ), and the maxScheduleSucc list is updated with the successors of schedule. At the point where the entire hollow schedules have finished execution, the values of maxPriority and maxScheduleSucc are updated as shown in Figure 4. For every newly-ready schedule, the conditions listed before will be evaluated. When schedule 8 is ready, it will not be considered as critical because it does not belong to the maxScheduleSucc list and its priority is not equal to max purple -1 .

Table I

Algorithm 1 The criticality counter with service delay

1: function ClusterScheduler

$\rightarrow$ select a ready cluster to execute

2: while IdleAllClusters do 

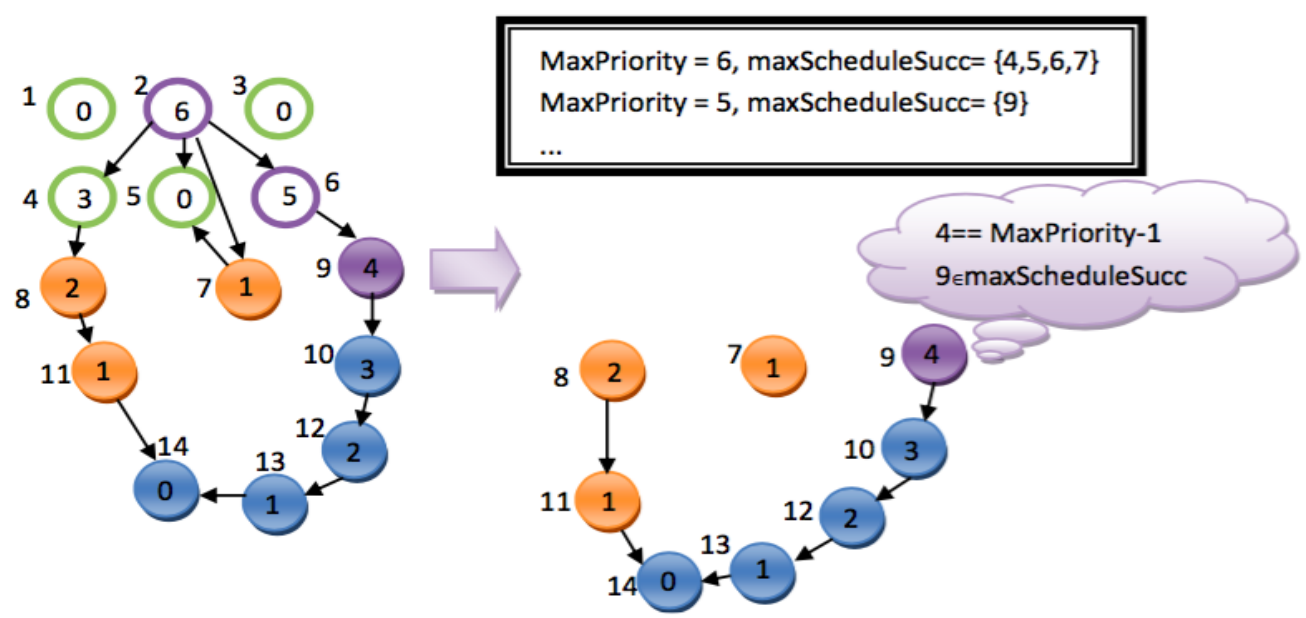

Figure 4. CAGSP execution. $\{1,2,3,4,5,6\}$ hollow nodes indicate finished schedules and pattern-purple nodes indicate critical schedules will be ready for execution with highest priority.

$\rightarrow \quad$ select clusters in the order based on the scheduling policy

3: $\quad \mathrm{w} \leftarrow$ findNextCluster $(1,2,3, \ldots, \mathrm{N}-1)$

4: if w.isRready()

then Delay time $=$ total delay time between two consecutive packets

5: $\quad$ w.nDelay $\leftarrow$ w.nDelay + Delay time $\quad(n=1,2, \ldots, C)$

6: SchedulerExecute(w)

7: end if

8: end while

9: end function

Table II

Algorithm 2 Update phase for link if $S_{l}^{D}(t)=1$

1: Choose a permutation $\left(\mathrm{n}_{1}^{l}, \mathrm{n}_{2}^{l}, \ldots, \mathrm{n}_{C}^{l}\right)$ of the set $\{1,2, \ldots, C\}$ according to criticality counter in Algorithm 1.

2: Let $f_{l}(x)=\exp \left(\alpha \mathrm{U}_{l}\left(\frac{x}{C}\right)\right)$ and $x_{l}^{1}(x)=\sum_{k=1}^{C} V_{l k}(\mathrm{t}-1)$

3: for $\mathrm{i}=1$ to $\mathrm{C}$ do

4: if $V_{l^{\prime}, n_{i}^{l}}(\mathrm{t}-1)=1$ for any link $l^{\prime} \in \varepsilon_{l}$ then

5: $V_{l, \mathrm{n}_{i}^{l}}(\mathrm{t})=V_{l, \mathrm{n}_{i}^{l}}(\mathrm{t}-1)$

6: else 
7: $\quad V_{l, \mathrm{n}_{i}^{l}}(\mathrm{t})$ is determined with CASP using Algorithm 5.

8: end if

9: $\quad x_{l}^{i+1}=x_{l}^{i}+V_{l, n_{i}^{l}}(t)-\mathrm{V}_{l, n_{i}^{l}}(\mathrm{t}-1)$

10: end for

11: Link $l$ broadcasts $\vec{V}_{l}(\mathrm{t})$ to all of its neighbors.

Table III

Algorithm 3 The Coordinated criticality-Aware Rate-function Acceleration algorithm (CARA)

1: Function AdmissionControlPredictor (rate, delay, throughput)

Select partition and compute maximum rate function by formula (29), minimum rate function by formula (33) and average rate of link $l$ is $r_{l}(\vec{V})=\mathrm{x}_{l}(\vec{V}) / \mathrm{C}$

2: Criticality-Aware Global Scheduler Priority return delay threshold.

3: VirtualChannel.delay $\leftarrow$ (VirtualChannelID, maximum rate function)

4: If ACP[VirtualChannel.delay] > Threshold then

5: Predicted to be critical schedule

6: CriticalSchedule.execution(channel ID)

7: set ACP access channel ID

8: else predicted to be non-critical cluster schedule

9: NonCriticalSchedule.access(channelID)

10: set ACP access channel ID

\section{1: end if}

\section{2: end function}

13: Function DynamicSchedulerClusterCriticalityPredictor (cluster ID, channel ID, maxpriority)

// Critical Cluster Identification with Criticality Prediction Logic (CPL)

Critical Schedule Prediction using Algorithm 4

14: If IsCriticalCluster(chan.ClusterID) then

15: correct prediction; increment ACP

16: ACP[VirtualChannel.delay]++ 
17: CSP[VirtualChannel.delay]++

18: else

19: schedule execution from non-critical cluster

20: CSP[VirtualChannel.delay]++

21: end if

22: end function

23: Function GreedyCriticality-AwareClusterScheduler (cluster ID, channel

ID, maxpriority) //Greedy Criticality-Aware Cluster Scheduler(GCACS)

24: if (Criticality[i]== Criticality $[\mathrm{i}-1])$

25: search oldest cluster schedule i-1

//Greedy-Then-Oldest algorithm

26: maxpriority $=$ priorityOf $(\mathrm{i}-1)$;

27: maxPrioritySchedule $=\mathrm{i}-1$;

28: end if

29: end function

30: Function Criticality-AwareVirtualMemoryPrioritization (cluster ID, channel ID, maxpriority)

//Criticality-Aware Global Scheduler Priority (CAGSP)

31: if (CSP. V==false) And

(CriticalSchedule.execution (channelID) $==$ CriticalSchedule $)$

Then incorrect prediction, schedule should be assigned to non-critical cluster;decrement ACP

32: ACP[VirtualChannel.delay]++

33: else if ((CSP. V==false) And

(CriticalSchedule.execution $($ channelID $)==$ NonCriticalSchedule $)$

34: then CSP[VirtualChannel.delay]++

35: end if

36: end function

Table IV

Algorithm 4 Critical Cluster Identification with Criticality Prediction Logic (CPL) 


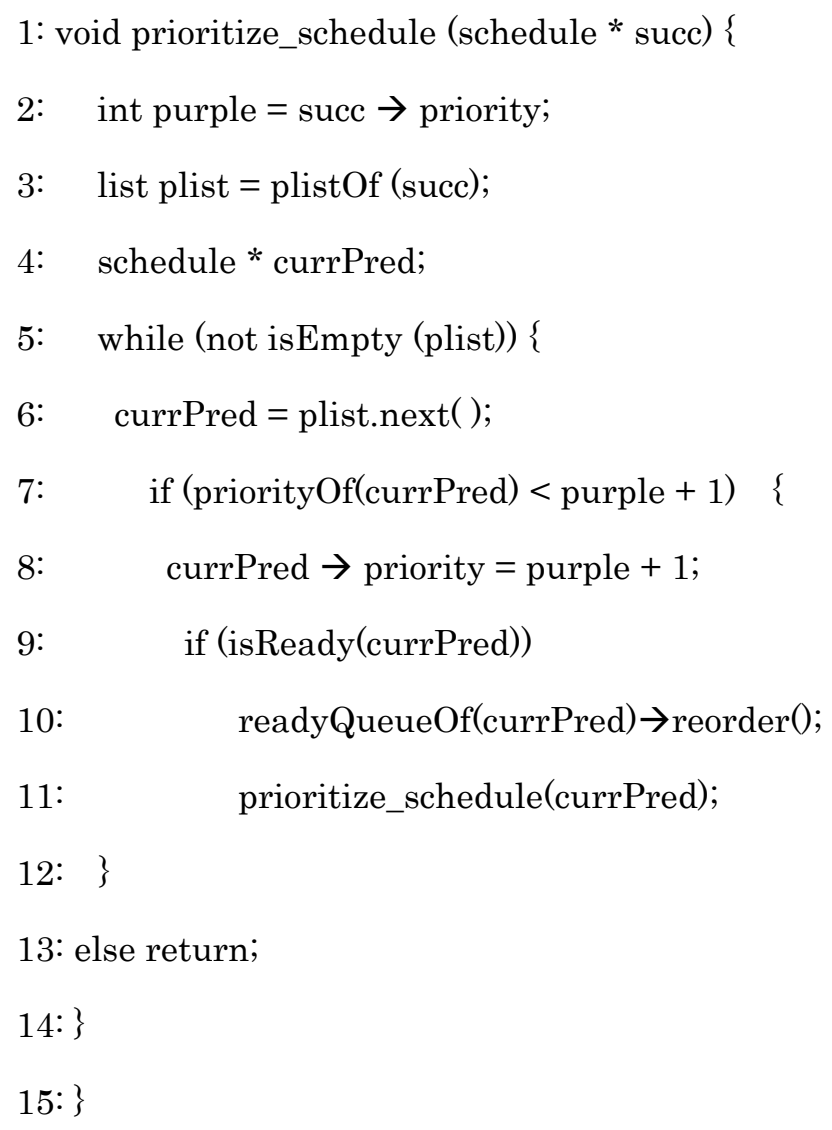




\section{PERFORMANCE MEASUREMENT}

Consider three performance metrics: 1) the throughput 2) the steady-state probability that the largest packet delay in the system exceeds a certain fixed threshold $b$, and 3) its rate-function in many-channel many-user regime.

Optimal throughput region [15] is defined as the union of the throughput regions of all possible scheduling policies, which is denoted by $\Lambda^{*}$. A scheduling policy is throughput-optimal if it can stabilize any arrival rate vector inside $\Lambda^{*}$. Achieve throughput-optimality in multichannel systems, each server choose a connected queue with a large enough weight (queue length or delay). The expected packet delay at link 1 must be equal to $1 / R_{l}$, which is upper-bounded by $\frac{1}{(1-\varepsilon) r_{l}^{\min }}$. A high throughput at link 1 refers to a low expected packet delay

$$
P\left\{d_{l}(t) \geq \mathrm{d}\right\} \leq\left(1-\mathrm{r}_{l}^{\min }\right)^{d}+\varepsilon
$$

where $d_{l}(t)$ is HOL waiting time.

$$
\text { Assume } 0<h<\frac{1}{(\Delta+2)(\mathrm{K}-1)} \text { and } \frac{\Delta_{l}+1}{C}<\frac{1}{K_{l}\left(\Delta_{l}+2\right)}-\frac{h\left(\mathrm{~K}_{l}-1\right)}{K_{l}}, \forall l,
$$

under CASP-VOMCP algorithm,

$$
\mathrm{r}_{l}^{\min } \geq \frac{1}{K_{l}\left(\Delta_{l}+2\right)}-\frac{h\left(\mathrm{~K}_{l}-1\right)}{K_{l}}-\frac{\Delta_{l}+1}{C} .
$$

If $0<h<\frac{1}{2(\Delta+2)(\mathrm{K}-1)}$ and $C \geq 4 K(\Delta+1)(\Delta+2)$, then $\mathrm{r}_{l}^{\min } \geq \frac{1}{4 K(\Delta+2)}$.

$\Delta_{l}=\left|\varepsilon_{l}\right|$ is the number of links in the neighborhood of link $1 . K_{l}$ is the maximum number of links that can be scheduled simultaneously in the neighborhood of link 1 .

When single-slot reconfiguration delay $T_{r}$, there is a frame length function

$$
x_{k}=T_{r}+\left(\sum_{i} Q_{i}\left(\mathrm{t}_{k}\right)\right)^{a}
$$

for a fixed $a \in(0,1)$, where $t_{k}$ is the first slot of the kth frame, $Q\left(\mathrm{t}_{k}\right)$ is the queue lengths at $t_{k}$.

Assuming that the system is stationary and ergodic, let $W(0)=\max _{1 \leq i \leq n} W_{i}(0)$ denote the largest HOL delay over all the queues (i.e., the largest packet delay in the system) in the steady state, then define rate-function 
$I(b)$ as the delay-rate of the probability that $W(0)$ exceeds any fixed integer threshold $b \geq 0$, as the system size $\mathrm{n}$ goes to infinity, i.e.,

$$
I(b)=\lim _{n \rightarrow \infty} \frac{-1}{n} \log _{2} P(\mathrm{~W}(0)>\mathrm{b}) .
$$

The estimate the delay-violation probability using $P(\mathrm{~W}(0)>\mathrm{b})=\exp (-\mathrm{nI}(\mathrm{b}))$ based on the knowledge of rate-function. With a large $\mathrm{n}$, a larger value of the rate-function implies a better delay performance, that is, a smaller probability that the largest packet delay exceeds a certain threshold. Optimal rate-function is defined as the maximum achievable ratefunction over all possible schedulers, which is denoted by $I^{*}(b)$. A scheduler is rate-function delay-optimal if it achieves the optimal rate-function $I^{*}(b)$ for any fixed integer threshold $b \geq 0$.

\subsection{Upper bound on the rate-function}

Let $\mathrm{A}_{i}(\mathrm{t})$ denote the number of packet arrivals to queue $Q_{i}$ in time slot t. Let $\mathrm{A}\left(\mathrm{t}_{1}, \mathrm{t}_{2}\right)=\sum_{\tau=t 1}^{t 2} \mathrm{~A}_{i}(\tau)$ denote the cumulative arrivals to the system from time $\mathrm{t} 1$ to time t2. Let $\lambda_{i}$ denote the mean arrival rate to queue $Q_{i}$ in time slot $\mathrm{t}$. let $\lambda=\left[\lambda_{1}, \lambda_{2}, \ldots, \lambda_{n}\right]$ denote the arrival rate vector. Let $I_{A G}(\mathrm{t}, \mathrm{x})$ denote the asymptotic decay-rate of the probability that in any interval of t time-slots, the total number of arrivals is greater than $\mathrm{n}(\mathrm{t}+\mathrm{x})$, as $\mathrm{n}$ tends to infinity, i.e.,

$$
I_{A G}(\mathrm{t}, \mathrm{x})=\lim \inf _{n \rightarrow \infty} \frac{-1}{n} \log _{2} P(\mathrm{~A}(-\mathrm{t}+1,0)>\mathrm{n}(\mathrm{t}+\mathrm{x}))
$$

Let $I_{A G}(\mathrm{x})$ be the infimum of $I_{A G}(\mathrm{t}, \mathrm{x})$ over all $t>0$, that is

$$
I_{A G}(\mathrm{x})=\inf _{t>0} I_{A G}(t, x) .
$$

Define $I_{X}=\log _{2}\left(\frac{1}{1-q}\right)$, for any scheduling algorithm, upper bound of ratefunction can be computed as follows:

$$
\begin{aligned}
& \lim \sup _{n \rightarrow \infty} \frac{-1}{n} \log _{2} P(\mathrm{~W}(0)>\mathrm{b}) \\
& \leq \min \left\{(\mathrm{b}+1) \mathrm{I}_{x}, \min _{0 \leq c \leq b}\left\{\mathrm{I}_{A G}(\mathrm{~b}-\mathrm{c})+\mathrm{cI}_{x}\right\}\right\}
\end{aligned}
$$




$$
=I_{U}(b) \text {. }
$$

$(b+1) I_{x}$ : the event that a queue with at least one packet is disconnected from

all of the $\mathrm{n}$ servers for consecutive $\mathrm{b}+1$ time-slots. $\mathrm{I}_{A G}(\mathrm{~b}-\mathrm{c})$ : the event that the arrivals are too bursty during the interval of $[-\mathrm{t}-\mathrm{b},-\mathrm{b}-1]$ such that at the beginning of time-slot $\mathrm{c}$ for $c \leq b$, there exists at least one packet remaining in the queue $\mathrm{Q} 1 . \mathrm{cI}_{x}$ : the event that the services are sluggish such that queue $\mathrm{Q} 1$ is disconnected from all of the $\mathrm{n}$ servers for the following consecutive c timeslots. Both of the above events will lead to the delay-violation event $\mathrm{W}(0)>\mathrm{b}$ under all the scheduling policies. $\mathrm{I}_{U}(b)$ is an upper bound on the rate-function that can be achieved by any scheduling policy. For the optimal rate-function $I^{*}(b), I^{*}(b) \leq \mathrm{I}_{U}($ b) for any fixed integer threshold $b \geq 0$. Optimal rate-function is $I^{*}(b)=(b+1) \log _{2}\left(\frac{1}{1-q}\right)$.

\subsection{Lower bound on the rate-function}

Lemma 1: For any scheduling algorithm, lower bound of rate-function can be computed as follows:

$$
\mathrm{I}_{L}(b)=\lim \inf _{n \rightarrow \infty} \frac{-1}{n} \log _{2} P(\mathrm{~W}(0)>\mathrm{b}) \geq \mathrm{I}^{*}(\mathrm{~b}-1)
$$

$I^{*}(b-1)$ : Optimal rate-function for threshold $b-1$.

$$
\begin{aligned}
& \mathrm{I}(b) \geq \mathrm{I}_{U}(\mathrm{~b}-1) \geq I^{*}(\mathrm{~b}-1), \\
& \mathrm{I}(b) \geq(\mathrm{b} /(\mathrm{b}+1)) \mathrm{I}^{*}(\mathrm{~b}),
\end{aligned}
$$

where $I^{*}(b)=(b+1) \log _{2}\left(\frac{1}{1-q}\right)$.

$$
\mathrm{I}_{L}(b)=\operatorname{blog}_{2}\left(\frac{1}{1-q}\right)
$$

\subsection{Utility Optimality}

This section discusses the capacity/utility performance in VMC-VOMCP algorithm.

The number of virtual channel $C>\frac{2 \log _{2} L}{3 \varepsilon^{2}}$ for any $\varepsilon \leq 0.1$ and L links. 
Global schedule $\vec{V}(\mathrm{t})$ as a Markov chain, $\mathrm{r}_{l}\left(\overrightarrow{\mathrm{V}}^{s}\right) \geq \mathrm{R}_{l}^{*}-\varepsilon$, for all link 1 .

$$
\begin{aligned}
& \mathrm{U}(\overrightarrow{\mathrm{r}})=\sum_{l=1}^{L} U_{l}\left(\mathrm{r}_{l}\right) \\
& P\left\{U(\vec{r}(\vec{V}(\mathrm{t}))) \geq \sum_{l=1}^{L} U_{l}\left(\left[\mathrm{R}_{l}^{*}-\varepsilon\right]^{+}\right)\right\} \geq 1-\varepsilon
\end{aligned}
$$

where $R_{l}^{*}$ is the optimal solution of problem (1).

The time-averaged throughput $\mathrm{R}_{l}$ of link $\mathrm{l}$ has a lower bound

$$
\mathrm{R}_{l} \geq(1-\varepsilon) \mathrm{r}_{l}^{\min } \cdot U(\vec{R}) \geq(1-\varepsilon) U\left(\left[\overrightarrow{\mathrm{R}}^{*}-\varepsilon\right]^{+}\right), \quad \text { where }
$$

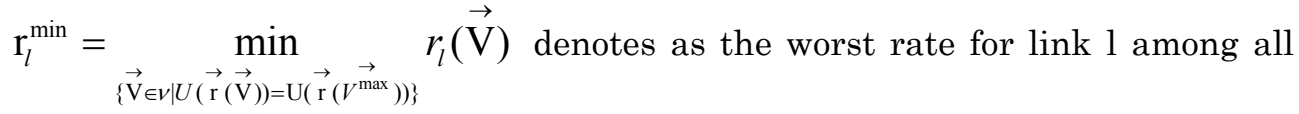
global schedules with the maximum utility.

\section{SIMULATION RESULTS}

Construct 23 wireless nodes deployed in a $900 \times 900 \mathrm{~m}^{2}$ 5G cellular network. The transmission range and interference range of each node is set to $900 \mathrm{~m}$ and $90 \mathrm{~m}$, respectively. Thus, $\mathrm{K}=10$. Peak data rate is $10 \mathrm{Gbps}$, minimal data rate is 100 Mbps. Latency is less than $1 \mathrm{~ms}$. 5G adopted full duplex cognitive radio transmitting signal at $60 \mathrm{GHz}$. Source node has 20 packets transmitted. Simulations for such $5 \mathrm{G}$ cellular network system are with $\mathrm{n}$ servers and $\mathrm{n}$ users, where $n \in\{10,20, \ldots, 100\}$. The simulation period lasts for $10^{9}$ time-slots for each policy. For channel model, assume ON-OFF channels with unit capacity, set $\mathrm{q}=0.75$. Run simulations in a Mac PC with Intel Core T5470 1.6 GHz CPU and 1GB memory. The weight is chosen as $w_{l}(\mathrm{t})=\log _{2}\left(0.5 \mathrm{Q}_{l}(\mathrm{t})\right)$. For CASPVOCMP, let $\mathrm{C}=30, \varepsilon=0.3$.

\subsection{Performance comparison of different scheduling policies with virtual channels}

Fig. 5 compares the performance of different scheduling policies in the case with virtual channels for delay threshold $b=8$. It demonstrates the probabilities that the largest HOL delay exceeds the threshold $b$ under VFMW, FBDC and CASPVOMCP scheduling schemes. VFMW has lower probability of delay than FBDC policy because of Max-Weight policy with adaptive frame sizes in VFMW scheme. As the number of secondary user increment considerably, the probability of delay becomes smaller. It has been shown that CASP-VOMCP empirically achieves near-optimal delay performance.

Fig. 6 plots the performance comparison of different scheduling policies in the case with virtual channels for 23 secondary users. It demonstrates the probabilities that the largest HOL delay exceeds the threshold b under D-SSG, 
VMC-CSMA and CASP-VOMCP scheduling schemes. VMC-CSMA has higher delay probability than D-SSG and CASP-VOMCP. CASP-VOMCP demonstrates the probability for different delay thresholds $b$ to investigate the performance of good policy for fixed users $n$.

\subsection{Performance evaluation of CASP-VOMP policy}

Fig. 7 shows the total utility of different delay parameter values under different virtual channels under CASP-VOMCP polices. Companied with the decrement of delay parameter a when it equals to 16 , total utility achieve an ideal performance. As delay parameter is 64 , there is a minimal utility performance. When the rate function is optimal, it can obtain the higher total utility. When the rate function is upper bound, total utility is larger than that of lower bound. When a is larger, the utility value after convergence is closer to the optimal value. Larger a incurs longer convergence time. $r_{l}=\frac{x_{l}^{i}}{C}$, as $\mathrm{C}$ increases, to maintain the same probability of adding another virtual channel, increase a proportional to $\mathrm{C}$.

Fig. 8 indicates the utility function of sum throughput under different reconfigurable delays with average queue length Qi=10 under CASP-VOMP policy. As the increment of reconfigurable delay, the sum throughput augments accordingly. When reconfigurable delay equals to $60 \mathrm{~s}$, it provides a good throughput-delay performance in CASP-VOMP policy. The larger number of virtual channels there are, the higher sum throughput it has.

Fig.9 illustrates the utility function of sum throughput under different average queue lengths with the change of reconfigurable delay. Due to the soaring of total average queue sizes, the sum-throughput grows up significantly. When reconfigurable delay augments, CASP-VOMP can achieve the optimal sum throughput.

Fig.10 presents the execution time of critical and non-critical clusters with various priorities in different virtual channels. Compared with (a) (b), it indicates that high priority schedule has smaller execution time than lower priority schedule. Meantime, it shows that critical cluster schedules run faster than noncritical clusters compared with (b) (c).

Fig.11 depicts the execution time of different virtual channels with different priorities. High priority schedule within higher delay queue runs faster than Low priority schedules compared with (b) (c). Low priority schedules wait in higher delay queue which require more execution time. High priority schedule within higher delay queue executes quickly than that of lower delay queue compared with (a) (b). 


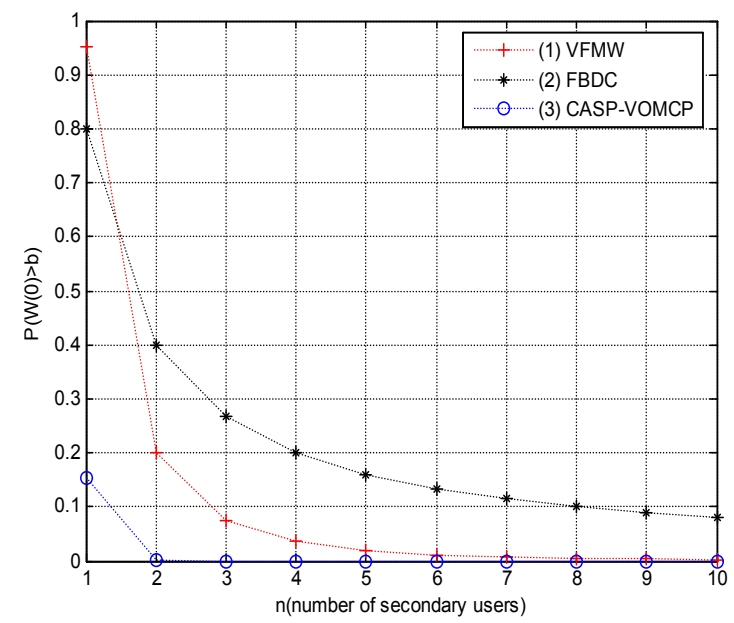

Fig.5 Delay probability of different scheduling polices with different number of secondary users for delay threshold $b=8$.

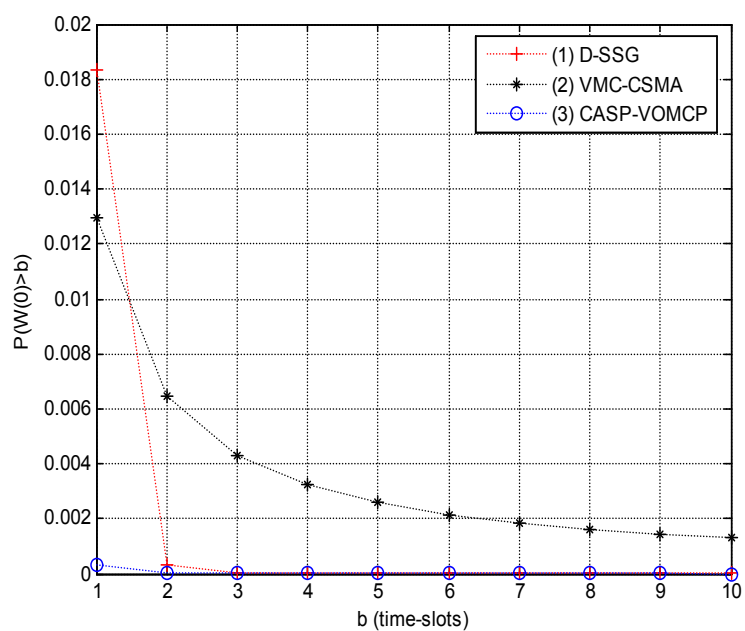

Fig.6 Delay probability of different scheduling polices with different delay thresholds for 23 secondary users.

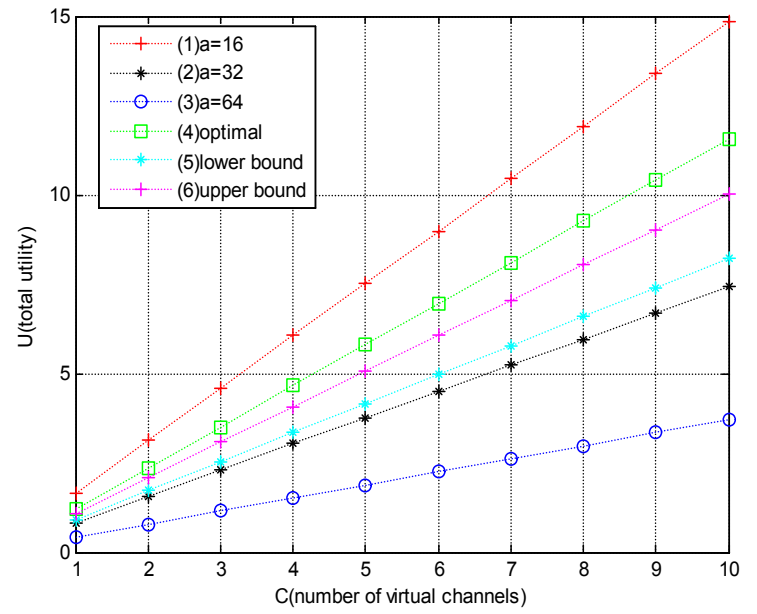

Fig.7 Total utility of different delay parameter values under different virtual channels in CASPVOMCP policy. 


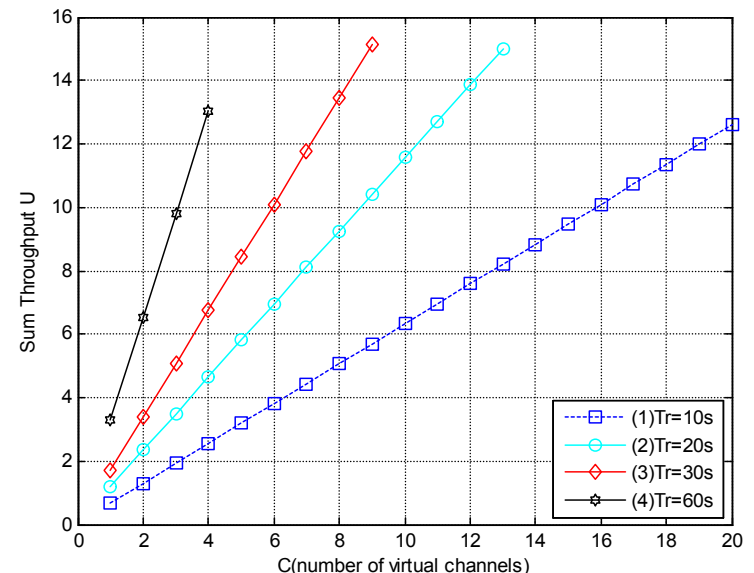

Fig.8 Utility function of sum throughput under different reconfigurable delays with average queue length Qi=10.

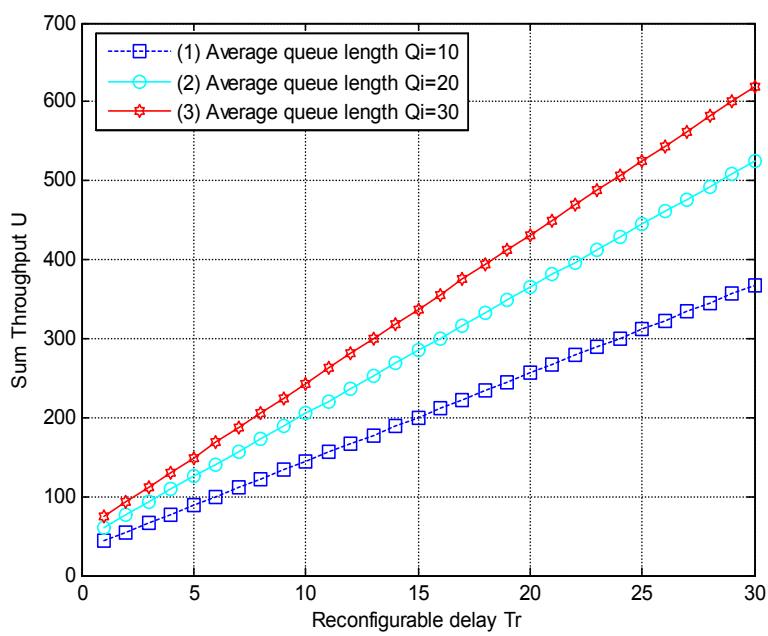

Fig.9 Utility function of sum throughput under different average queue lengths with the change of reconfigurable delay

Fig.12 compares that the execution time with different priority schedules in critical cluster and non-critical cluster. When high priority schedule in noncritical cluster occupies 8 to 18 virtual channels, it can run the same speed among the critical cluster and non-critical cluster, high priority schedule and low priority.

Fig.13 elaborates that criticality with different priority schedules in critical cluster and non-critical cluster. High priority schedule in critical cluster has highest criticality. As the number of virtual channels soars up, the criticality plumps correspondingly. Low priority schedule in critical cluster runs faster than high priority schedule in non-critical cluster.

Fig.14 indicates that the execution time of different priority schedules within critical cluster and non-critical cluster over virtual channels. High priority schedule within critical cluster requires longer execution time than low priority schedule within critical cluster compares with (a) (c). Non-critical cluster schedules need smaller execution time than critical cluster schedules compared with (a) (b).

Fig. 15 demonstrates that criticality of critical cluster and non-critical cluster's scheduling priority over virtual channels. High priority schedule within 
critical cluster has larger criticality than low priority schedule within critical cluster compared with (a) (c). Critical cluster schedules can achieve more optimal criticality performance than non-critical cluster schedules compared with (a) (b).

Fig. 16 shows that criticality prediction accuracy of CARA with CSP and $\mathrm{ACP}$ at maximum rate, average rate function and minimum rate function. In light of the increment of reconfigurable delay, criticality prediction accuracy approaches optimal. When CSP implements with maximum rate, criticality prediction accuracy is the highest. The criticality prediction accuracy of CSP is better than that of ACP. The higher speed it executes, the more accuracy the predictor has.

Fig. 17 presents that execution time improvement of CARA with CSP and $\mathrm{ACP}$ at maximum rate, average rate function, and minimum rate function. CSP and $\mathrm{ACP}$ have a similar distribution in execution time. ACP at maximum rate requires highest execution time than that of average rate function and minimum rate function. CSP runs faster than ASP at minimum rate function.

Fig. 18 elaborates that the throughput improvement of $\mathrm{CARA}_{\mathrm{CPL}}$,

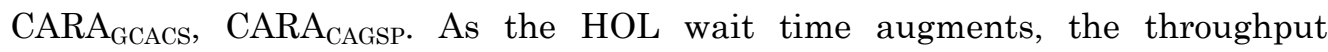
improvement of CARA soars up considerable. GCACS can enhance more throughput performance than CPL. CAGSP improves slightly than CPL and GCACS in sum throughput.

Fig. 19 depicts that the HOL waiting time of $\mathrm{CARA}_{\mathrm{CPL}}, \mathrm{CARA}_{\mathrm{GCACS}}$, CARA $_{\text {CAGSP. }}$ As delay threshold increases, CARA requires more HOL wait time. CAGSP has largest wait time than GCACS and CPL. CPL has lowest HOL wait time than others.

Fig. 20 compares that the execution time improvement of $\mathrm{CARA}_{\mathrm{CPL}}$,

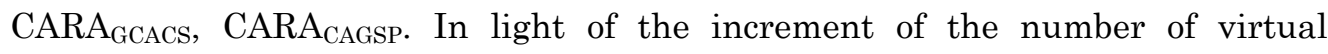
channels, for a fixed value of threshold $\mathrm{b}$ to compare the execution time enhancement of CARA, execution time improvement of CARA grows up. Compared with GCACS and CPL, CAGSP has largest performance optimization which has more superiority in CASP-VOMCP policy.

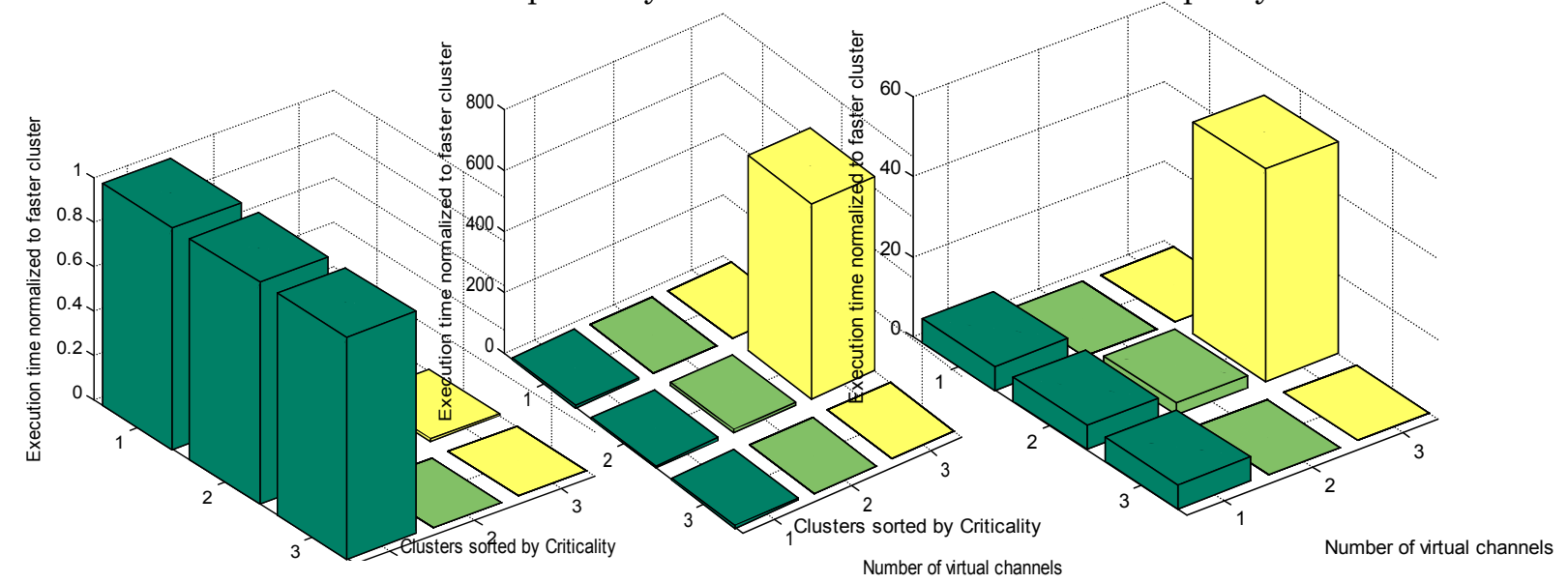



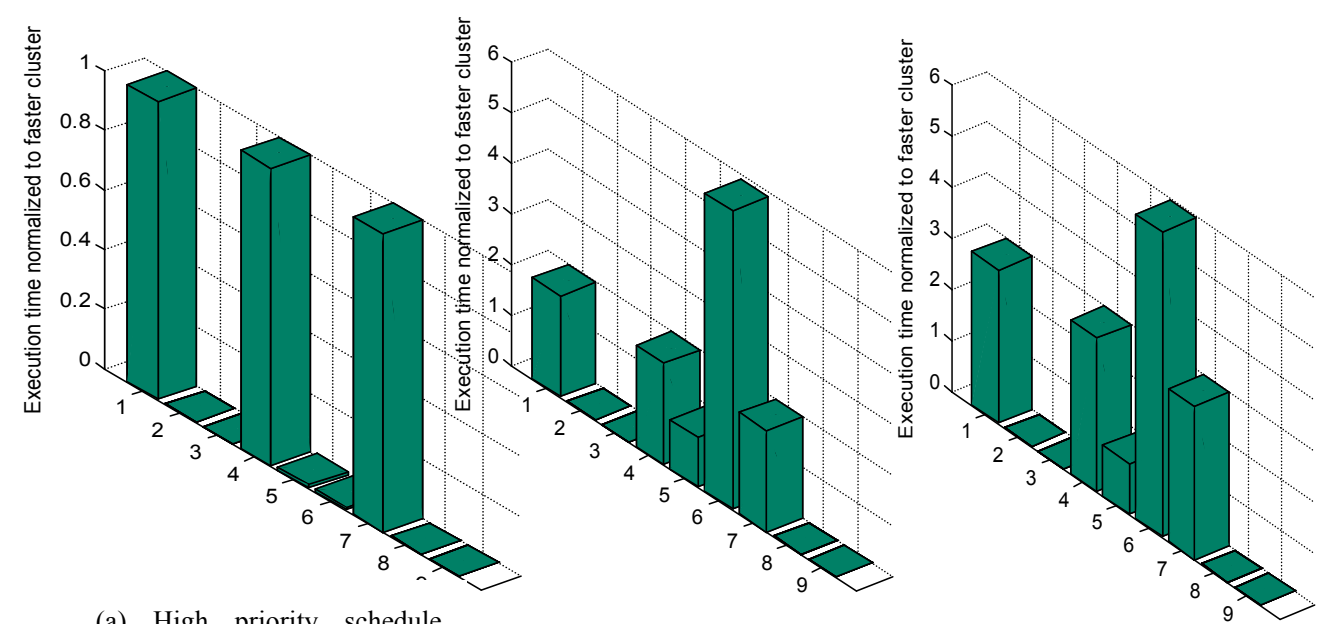

(a) High priority schedule

(b) High priority schedule within higher delay queue

Number of virtual channels

within lower delay queue

Fig.11 Comparison of execution time of different virtual channels $\quad$ (c) Low priority schedule within higher delay queue
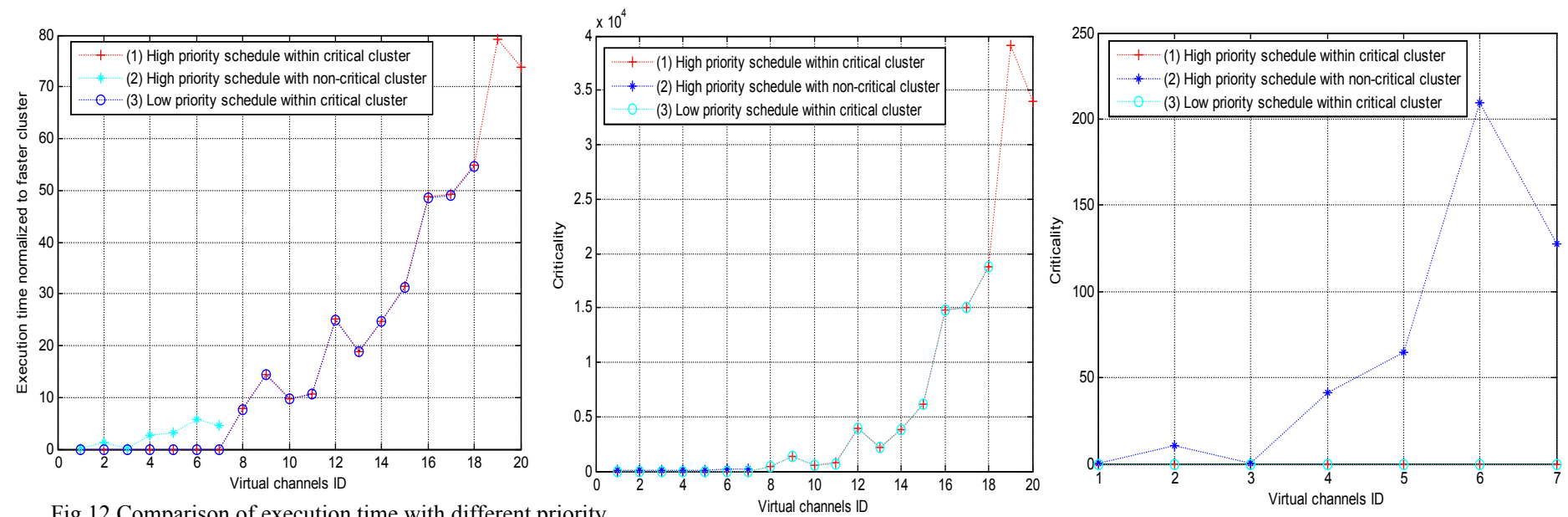

Fig.12 Comparison of execution time with different priority

Virtual channels ID

schedules in critical cluster and non-critical cluster

Fig.13 Comparison of criticality with different priority schedules in critical cluster and non-critical cluster

(a) High priority schedule within critical cluster

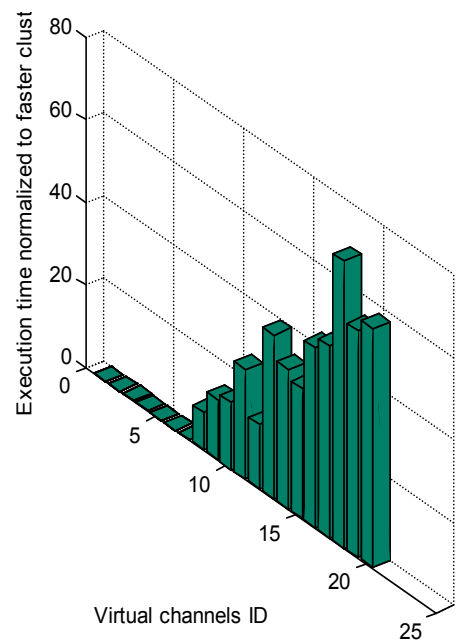

(c) Low priority schedule within critical cluster

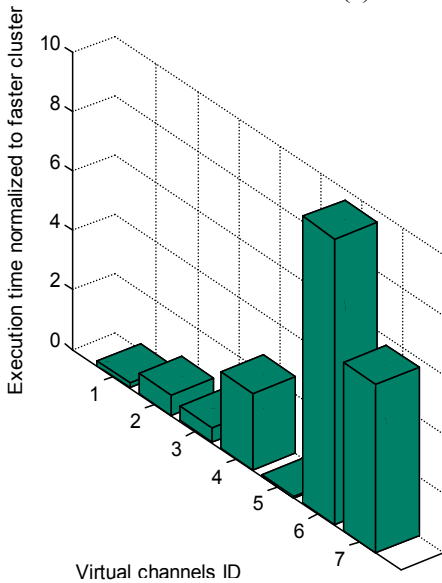

(b) High priority schedule within non-critical cluster

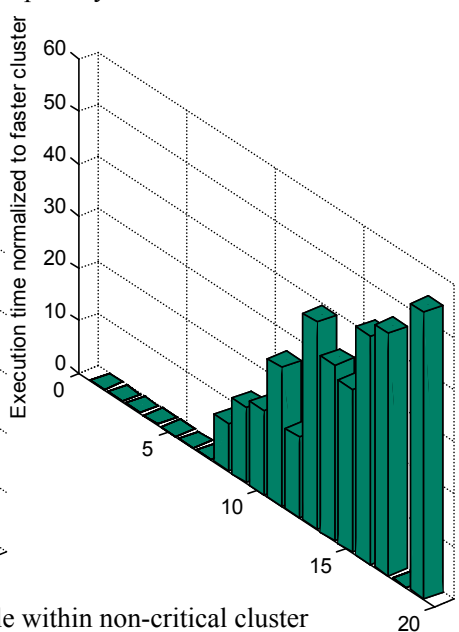

Fig.14 Execution time of different priority schedules within critical cluster and non-critical cluster over virtual channels. 


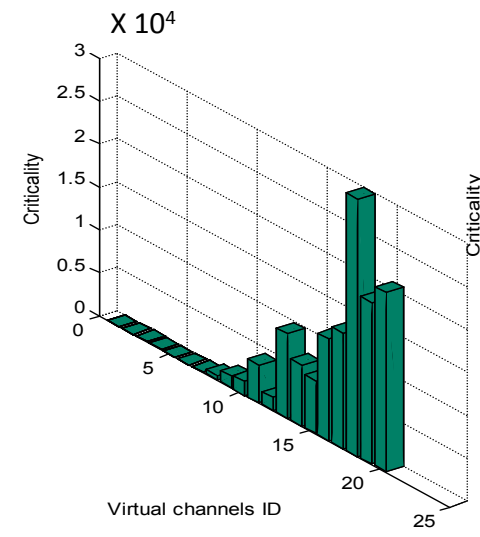

(a) High priority schedule within critical cluster

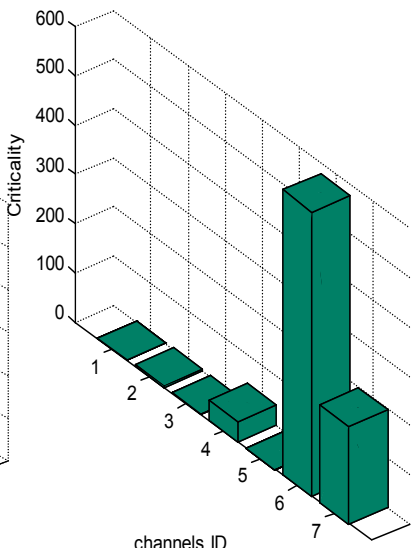

channels ID

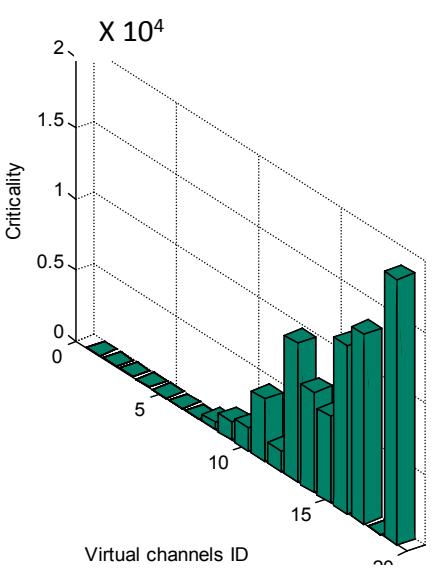

Virtual channels ID

(b) High priority schedule within non-critical cluster

(c) Low priority schedule within critical cluster Fig.15 Criticality of critical cluster and non־critical cluster's scheduling priority over virtual channels.

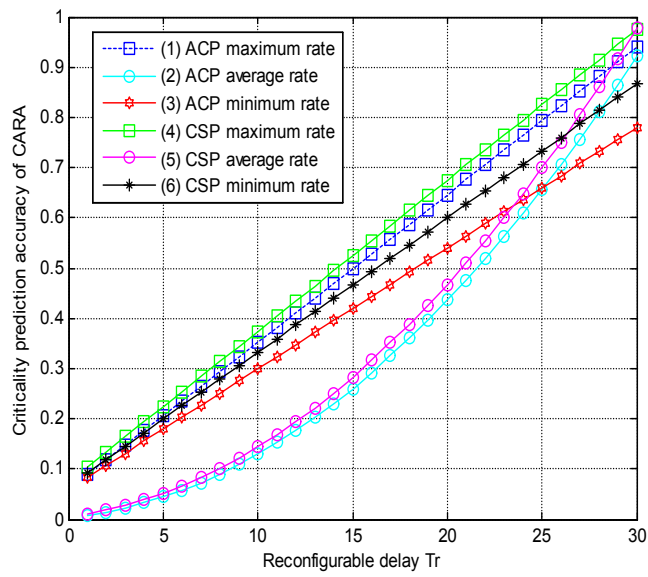

Fig.16 The criticality prediction accuracy of CARA with CSP and ACP at maximum rate and average rate function

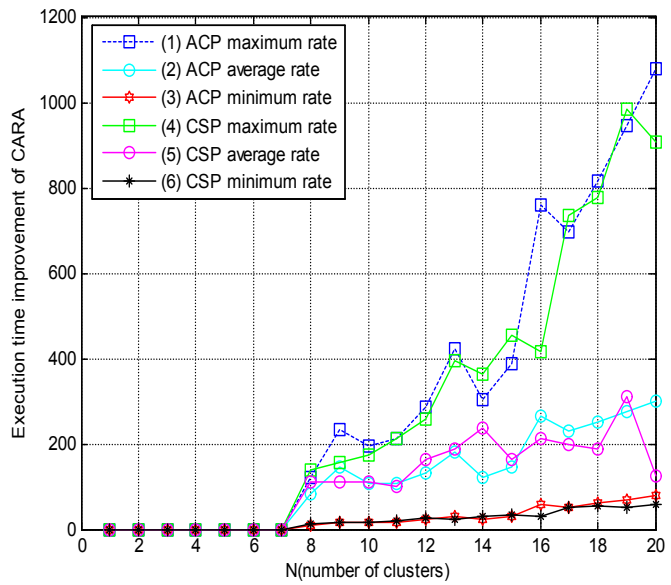

Fig.17 The execution time improvement of CARA with CSP and ACP at maximum rate and average rate function 


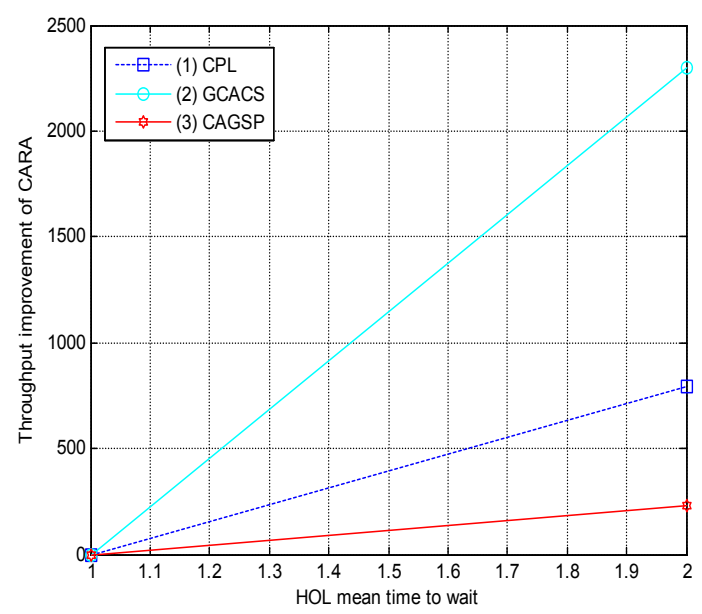

Fig.18 The throughput improvement of $\mathrm{CARA}_{\mathrm{CPL}}, \mathrm{CARA}_{\mathrm{GCACS},} \mathrm{CARA}_{\mathrm{CAG}}$

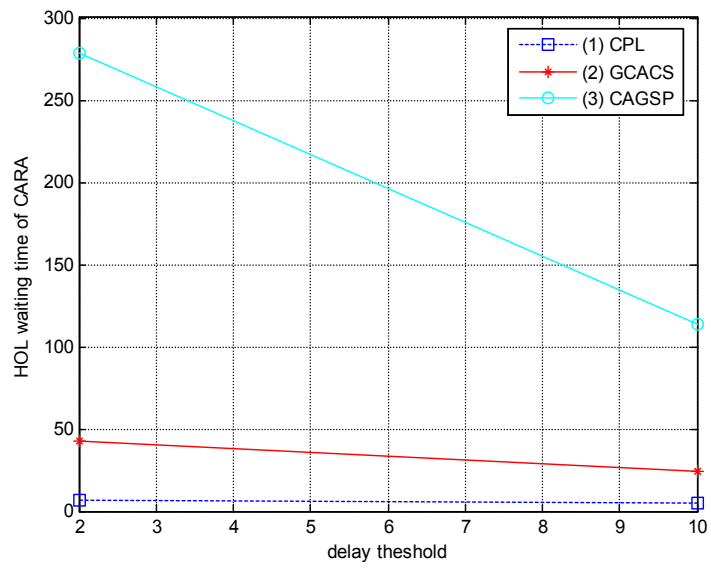

Fig.19 The HOL waiting time of $\mathrm{CARA}_{\mathrm{CPL}}, \mathrm{CARA}_{\mathrm{GCACS}}$, $\mathrm{CARA}_{\mathrm{CAGSP}}$

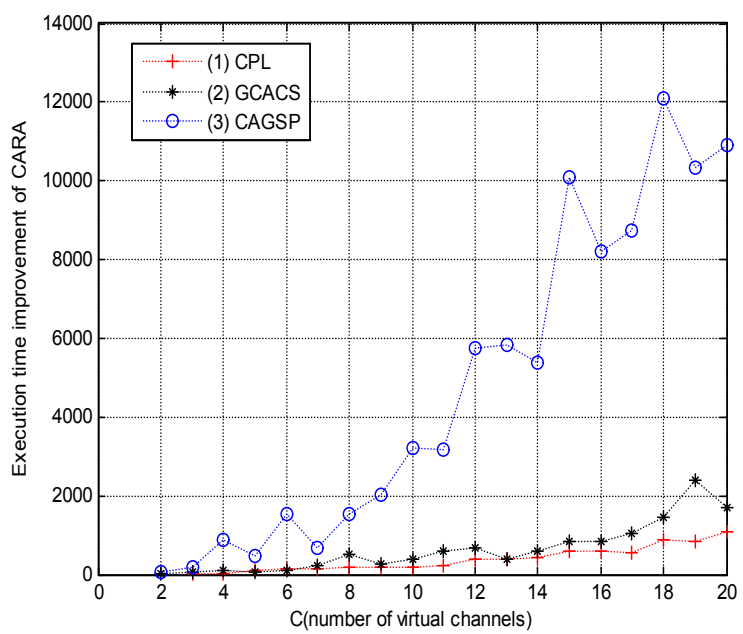

Fig.20 The execution time improvement of $\mathrm{CARA}_{\mathrm{CPL}}, \mathrm{CARA}_{\mathrm{GCACS}}, \mathrm{CARA}_{\mathrm{CAGSP}}$ 


\section{CONCLUSION}

This paper summarizes the current state-of-art technical solutions for $5 \mathrm{G}$ communication. The most significant contribution is to develop system model, algorithm implementation of CASP-VOMCP, CARA as well as performance metrics of proposed CASP-VOMCP, then to derive a lower bound on the rate-function that can be achieved by any scheduling policy. Furthermore, conduct numerical simulations and present results on throughput optimality and near-optimal ratefunction for low-complexity greedy policy.

\section{REFERENCES}

[1].Erik Dahlman et al., "5G Wireless Access: Requirements and Realization," IEEE Commun. Mag., vol.52, no.12, Dec. 2014, pp.42-47.

[2].M.Zubair Shafiq, Lusheng Ji, Alex X.Liu, Jeffrey Pang, and Jia Wang, "Large-Scale Measurement and Characterization of Cellualr Machine-to-Machine Traffic”, IEEE/ACM Trans. Netw., vol.21, no.6, Dec.2013, pp.1960-1973.

[3].Shunqing Zhang, Xiuqiang Xu, Yiqun Wu and Lei Lu, "5G: Towards Energy-Efficient, Low-Latency and High-Reliable Communications Networks," ICCS, 2014, pp.197-201.

[4].Shunqing Zhang, Xiuqiang Xu, Lei Lu, Yiqun Wu,Gaoning He, and Yan Chen, "Sparse Code Multiple Access: An Energy Efficient Uplink Approach for 5 G wireless Systems,"Globecom2014, Wireless Networking Symposium, pp.4782-4787.

[5].Peng Wang,Yonghui Li, Lingyang Song, and Branka Vucetic, "Multi-Gigabit Millimeter Wave Wireless Communications for 5G: From Fixed Access to Cellular Networks," IEEE Commun. Mag., vol.53, no.1, Jan. 2015, pp.168-178.

[6].Lili Wei, Rose Qingyang Hu,Yi Qian, Geng Wu, "Key Elements to Enable Millimeter Wave Communications for 5G Wireless Systems," IEEE Wireless Commun., vol. 21, no. 6, Dec. 2014, pp.136-143. [7].Jeffrey G.Andrews et al., "What Will 5G Be?" IEEE Journal on Selected Areas in Communications, vol.32, no.6, Jun. 2014, pp.1065-1082.

[8].Cheng-Xiang Wang et al., "Cellular Architecture and Key Technologies for 5G Wireless Communication Networks," IEEE Commun. Mag., vol.52, no.2, Feb. 2014, pp.122-130.

[9].Rose Qingyang Hu and Yi Qian, "An Energy Efficient and Spectrum Efficient Wireless Heterogeneous Network Framework for 5G Systems," IEEE Commun. Mag., vol.52, no.5, May 2014, pp.94-101.

[10].Shuangfeng Han, Chih-Lin I, Zhikun Xu, Chengkang Pan, Zhengang Pan, "Full Duplex: Coming into Reality in 2020?” Globecom2014, Wireless Networking Symposium, pp.4776-4781.

[11].Yu Cheng et al., "A Systematic Study of Maximal Scheduling Algorithms in Multiradio Multichannel Wireless Networks," IEEE/ACM Trans. Netw., pp.1-14, Jun.2014.

[12].Güner D. Çelik, and Eytan Modiano, "Scheduling in Networks with Time-varying channels and reconfiguration delay," IEEE/ACM Trans. Netw., vol.23, no.1, Feb.2015, pp.99-113.

[13].Reuven Cohen, Guy Grebla, "Joint Scheduling and Fast Cell Selection in OFDMA Wireless Networks," IEEE/ACM Trans. Netw., vol.23, no.1, Feb.2015, pp.114-125.

[14].Kang Chen, and Haiying Shen, "DTN-FLOW: Inter-Landmark Data Flow for High-Throughput Routing in DTNs," IEEE/ACM Trans. Netw., vol.23, no.1, Feb.2015, pp.212-226. 
[15].Bo Ji, Gagan R. Gupta, Manu Sharma, Xiaojun Lin, and Ness B.Shroff, "Achieving optimal throughput and near-optimal asymptotic delay performance in Multichannel wireless networks with low complexity: A practical greedy scheduling policy," IEEE/ACM Trans. Netw., vol.23, no.3, Jun.2015, pp.880893.

[16].Po-Kai Huang, Xiaojun Lin, "Achieving optimal throughput utility and low delay with CSMA-Like Algorithms: A Virtual Multichannel Approach,” IEEE/ACM Trans. Netw., vol.23, no.2, April.2015, pp.505518.

[17].Didem Gözüpek, Mordechai Shalom, and Fatih Alagöz, “A Graph-Theoretic Approach to Scheduling in Cognitive Radio Networks," IEEE/ACM Trans. Netw., vol.23, no.1, Feb.2015, pp.317-328.

[18].Jiliang Wang, Wei Dong, Zhichao Cao, and Yunhao Liu, "On the Delay Performance in a Large-Scale Wireless Sensor Network: Measurement, Analysis, and Implications," IEEE/ACM Trans. Netw., vol.23, no.1, Feb.2015, pp.186-197.

[19].Hu Jin, Bang Chul Jung,and Victor C. M. Leung, "Fundamental Limits of CDF-Based Scheduling: Throughput, Fairness, and Feedback Overhead," IEEE/ACM Trans. Netw., vol.23, no.3, June.2015, pp.894-907.

[20].Shin-Ying Lee, Akhil Arunkumar, and Carole-Jean Wu, "CAWA: Coordinated Warp Scheduling and Cache Prioritization for Critical Warp Acceleration of GPGPU Workloads," The $42^{\text {nd }}$ IEEE International Conference on Computer Architecture, ISCA'15, June 13-17, 2015, Portland, OR, USA.

[21].Lavanya Subramanian, Donghyuk Lee, Vivek Seshadri, Harsha Rastogi, Onur Mutlu, "The Blacklisting Memory Scheduler: Achieving High Performance and Fairness at Low Cost,” ICCD'14, pp.1-8, USA.

[22].Kallia Chronaki, Alejandro Rico, Rosa M. Badia, Eduard Ayguadé, Jesús Labarta, Mateo Valero, “Criticality-Aware Dynamic Task Scheduling for Heterogeneous Architectures," ICS'15, June 8-11, 2015, Newport Beach, CA, USA.

[23].Reetuparna Das, Onur Mutlu, Thomas Moscibroda, Chita R. Das, "Application-Aware Prioirization Mechanisms for On-Chip Networks," MICRO’09, December 12-16, 2009, New York, NY, USA.

Dan Ye has been working towards the Ph.D. degree at the Department of Computer Science and Information Engineering, National Taiwan University. Her research interests include cognitive radio systems, wireless communications, mobile computing, routing protocol, wireless sensor networks, distributed maximal scheduling algorithm, LTE networks, 5 G cellular network, next-generation Internet architectures, automatic control, advanced compiler, operating system, multi-core processor. 